\title{
A class of conformal mappings with applications to function spaces
}

\author{
Daniel Girela
}

\author{
Dedicated to Professor Joseph Cima on the occasion of his 70th birthday
}

\begin{abstract}
We discuss some of the properties of a class of conformal mappings introduced in a joint work with O. Blasco and M. A. Márquez and show that they can be used to prove the sharpness of a number of embedding theorems between certain spaces of analytic functions and, also, to prove that, for $1 \leq$ $p<\infty$, the space $\mathcal{B} \cap H^{p}$ (where $\mathcal{B}$ is the Bloch space) does not have the so called $f$-property of Havin.
\end{abstract}

\section{Some results on conformal mappings}

1.1. Basic notation. Let $\mathbb{D}$ denote the open unit disk of the complex plane $\mathbb{C}$. If $0<r<1$ and $f$ is an analytic function in $\mathbb{D}$ (abbreviated $f \in H(\mathbb{D})$ ) we set

$$
\begin{gathered}
M_{p}(r, f)=\left(\frac{1}{2 \pi} \int_{0}^{2 \pi}\left|f\left(r e^{i t}\right)\right|^{p} d t\right)^{1 / p}, \quad I_{p}(r, f)=M_{p}^{p}(r, f),(0<p<\infty), \\
M_{\infty}(r, f)=\sup _{0 \leq t \leq 2 \pi}\left|f\left(r e^{i t}\right)\right| .
\end{gathered}
$$

For $0<p \leq \infty$ the Hardy space $H^{p}$ consists of those functions $f \in H(\mathbb{D})$ for which $\|f\|_{H^{p}} \stackrel{\text { def }}{=} \sup _{0<r<1} M_{p}(r, f)<\infty$. We refer to [9] for the theory of Hardy spaces. The space $B M O A$ consists of those functions $f \in H^{1}$ whose boundary values have bounded mean oscillation on $\mathbb{T}=\partial \mathbb{D}$ (cf. [4], [11] and [14]).

2000 Mathematics Subject Classification. Primary 30C20, 30D45, 30D55; Secondary 30C35, $30 \mathrm{D} 50$.

Key words and phrases. Conformal mapping, Starlike domain, Circularly symmetric domain, Bloch functions, Normal functions, Mean Lipschitz spaces, Hardy spaces, Division by inner functions.

This research has been supported in part by grants from el Ministerio de Educación y Ciencia, Spain (MTM2004-00078 and MTM2004-21420-E) and by a grant from "La Junta de Andalucía" (FQM-210). 
1.2. On the relation between $M_{\infty}(r, F)$ and $M_{1}\left(r, F^{\prime}\right)$ when $F$ is a conformal mapping from the unit disk onto a certain domain. Let $\Omega$ be a simply connected domain contained in $\mathbb{C}$ with $0 \in \Omega \neq \mathbb{C}$ and let $F$ be the conformal mapping from $\mathbb{D}$ onto $\Omega$ with $F(0)=0$ and $F^{\prime}(0)>0$. We note that, for $0<r<1$, the quantity $2 \pi r M_{1}\left(r, F^{\prime}\right)$ represents the length of the closed curve $C_{r}$ which is the image under $F$ of the circle $\{|z|=r\}$. Clearly, we have that $M_{\infty}(r, F) \leq \operatorname{length}\left(C_{r}\right), 0<r<1$, and, hence,

$$
M_{\infty}(r, F)=\mathrm{O}\left(M_{1}\left(r, F^{\prime}\right)\right), \quad \text { as } r \rightarrow 1 .
$$

It is natural to look for a geometric condition on the domain $\Omega$ which is sufficient to imply that the quantities $M_{1}\left(r, F^{\prime}\right)$ and $M_{\infty}(r, F)$ are comparable. Keogh [24] and Hayman $[\mathbf{2 2}]$ proved that being starlike with respect to the origin is not such a sufficient condition.

1.3. Circularly symmetric domains. A domain $\Omega$ in $\mathbb{C}$ is said to be circularly symmetric if, for every $r$ with $0<r<\infty, \Omega \cap\{|z|=r\}$ is either empty, is the whole circle $|z|=r$, or is a single arc on $|z|=r$ which contains $z=r$ and is symmetric with respect to the real axis.

Let $\Omega$ be a simply connected and circularly symmetric domain in $\mathbb{C}$ with $\Omega \neq \mathbb{C}$ and $0 \in \Omega$. Let $F$ be the conformal mapping from $\mathbb{D}$ onto $\Omega$ with $F(0)=0$ and $F^{\prime}(0)>0$. Then (see [23] or the Corollary in p. 154 of $[\mathbf{3}]$ ) it is known that:

(a) $M_{\infty}(r, F)=F(r), 0<r<1$.

(b) For every $r$ with $0<r<1,\left|F\left(r e^{i \theta}\right)\right|$ is a decreasing function of $\theta$ in $[0, \pi]$.

1.4. The geometric condition. In the following theorem, which was obtained in a joint work with $\mathrm{O}$. Blasco and $\mathrm{M}$. A. Márquez [5], we give a geometric condition on $\Omega$ which implies that $M_{\infty}(r, F) \asymp M_{1}\left(r, F^{\prime}\right)$, as desired.

Theorem 1.1. ([5, Theorem 3.1]). Let $\Omega$ be a domain in $\mathbb{C}$ with $0 \in \Omega$ and $\Omega \neq \mathbb{C}$. Suppose that $\Omega$ is both circularly symmetric and starlike with respect to 0 . Let $F$ be the conformal mapping from $\mathbb{D}$ onto $\Omega$ with $F(0)=0$ and $F^{\prime}(0)>0$. Then there exists a positive constant $C$ such that

$$
2 \pi r M_{1}\left(r, F^{\prime}\right) \leq C M_{\infty}(r, F), \quad 0<r<1 .
$$

Consequently,

$$
M_{\infty}(r, F) \asymp M_{1}\left(r, F^{\prime}\right), \quad \text { as } r \rightarrow 1 .
$$

To prove Theorem 1.1 we use (b) together with the well known fact that (see p. 43 of [29] or p. 41 of $[\mathbf{1 0}])$, since $\Omega$ is starlike with respect to the origin, for every $r$ with $0<r<1, \arg F\left(r e^{i \theta}\right)$ is an increasing function of $\theta$ in $[0,2 \pi]$.

1.5. A special type of domain. In this section we consider a certain family of domains which satisfy the conditions of Theorem 1.1 and which have been useful to solve a number of problems in the theory of spaces of analytic functions.

Let $\Gamma=\left\{\gamma_{n}\right\}_{n=1}^{\infty}$ be a decreasing sequence of positive numbers with $\gamma_{1}<1$. We set

$$
\Omega_{\Gamma}=\Omega_{\left\{\gamma_{n}\right\}}=\mathbb{D} \cup\left(\bigcup_{n=1}^{\infty}\left\{z:|\operatorname{Im} z|<\gamma_{n}, \operatorname{Re} z>0, n \leq|z|<n+1\right\}\right) .
$$


It is clear that the domain $\Omega_{\Gamma}$ is circularly symmetric and starlike with respect to the origin. Consequently, if $F_{\Gamma}$ is the conformal mapping from $\mathbb{D}$ onto $\Omega_{\Gamma}$ with $F_{\Gamma}(0)=0$ and $F_{\Gamma}^{\prime}(0)>0$, we have that:

(i) $M_{\infty}\left(r, F_{\Gamma}\right)=F_{\Gamma}(r), 0<r<1$.

(ii) $M_{\infty}\left(r, F_{\Gamma}\right) \asymp M_{1}\left(r, F_{\Gamma}^{\prime}\right)$, as $r \rightarrow 1$.

Furthermore, $\left|\operatorname{Im} F_{\Gamma}\right|$ is bounded and, hence

(iii) $F_{\Gamma} \in B M O A$.

Using the Carathéodory kernel convergence theorem (cf. [29, pp. 28-31]), we can prove the following result (see the proof of Theorem 3.2 of [5] for the details).

TheOrem 1.2. Let $\varphi:[0,1) \rightarrow[0, \infty)$ be a function with $\varphi(r) \rightarrow \infty$ as $r \rightarrow 1$. Then there exists a decreasing sequence of positive numbers $\Gamma=\left\{\gamma_{n}\right\}_{n=1}^{\infty}$ with $\gamma_{1}<1$ such that, if $F_{\Gamma}$ is the conformal mapping from $\mathbb{D}$ onto $\Omega_{\Gamma}$ with $F_{\Gamma}(0)=0$ and $F_{\Gamma}^{\prime}(0)>0$, then we have that $F_{\Gamma}(r) \rightarrow \infty$, as $r \rightarrow 1$ and

$$
M_{\infty}\left(r, F_{\Gamma}\right)=F_{\Gamma}(r) \leq \varphi(r), \quad 0<r<1 .
$$

\section{On the sharpness of certain embedding theorems}

2.1. Bloch functions and normal functions. We recall that a function $f$ analytic in $\mathbb{D}$ is a Bloch function if

$$
\sup _{z \in \mathbb{D}}\left(1-|z|^{2}\right)\left|f^{\prime}(z)\right|<\infty .
$$

The space of all Bloch functions is denoted by $\mathcal{B}$. It is well known that

$$
H^{\infty} \subset B M O A \subset \mathcal{B} \text {. }
$$

The little Bloch space $\mathcal{B}_{0}$ consists of those functions $f$ which are analytic in $\mathbb{D}$ and satisfy

$$
\lim _{|z| \rightarrow 1}\left(1-|z|^{2}\right)\left|f^{\prime}(z)\right|=0 \text {. }
$$

The space $\mathcal{B}_{0}$ is a closed subspace of the Bloch space. In fact, $\mathcal{B}_{0}$ is the closure of the polynomials in the Bloch norm. We mention [2] as a general reference for the theory of Bloch functions

A function $f$ which is meromorphic in $\mathbb{D}$ is said to be a normal function if

$$
\sup _{z \in \mathbb{D}}\left(1-|z|^{2}\right) \frac{\left|f^{\prime}(z)\right|}{1+|f(z)|^{2}}<\infty .
$$

We refer to $[\mathbf{2}]$ and $[\mathbf{2 9}]$ for the theory of normal functions. For simplicity, let $\mathcal{N}$ denote the set of all holomorphic normal functions in $\mathbb{D}$. Any Bloch function is a normal function, that is, $\mathcal{B} \subset \mathcal{N}$.

2.2. Mean Lipschitz spaces of analytic functions. If $f$ is a function which is analytic in $\mathbb{D}$ and has a non-tangential limit $f\left(e^{i \theta}\right)$ at almost every $e^{i \theta} \in \mathbb{T}$, we define

$$
\begin{gathered}
\omega_{p}(\delta, f)=\sup _{0<|t| \leq \delta}\left(\frac{1}{2 \pi} \int_{-\pi}^{\pi}\left|f\left(e^{i(\theta+t)}\right)-f\left(e^{i \theta}\right)\right|^{p} d \theta\right)^{1 / p}, \quad \delta>0, \quad \text { if } 1 \leq p<\infty, \\
\omega_{\infty}(\delta, f)=\sup _{0<|t| \leq \delta}\left(\operatorname{ess.sup}_{\theta \in[-\pi, \pi]}\left|f\left(e^{i(\theta+t)}\right)-f\left(e^{i \theta}\right)\right|\right), \quad \delta>0 .
\end{gathered}
$$


Then $\omega_{p}(., f)$ is the integral modulus of continuity of order $p$ of the boundary values $f\left(e^{i \theta}\right)$ of $f$.

Throughout the paper $\omega:[0,1] \rightarrow[0, \infty)$ will be a continuous and increasing function with $\omega(0)=0$. Then, for $1 \leq p \leq \infty$, the mean Lipschitz space $\Lambda(p, \omega)$ consists of those functions $f \in H^{p}$ which satisfy

$$
\omega_{p}(\delta, f)=\mathrm{O}(\omega(\delta)), \quad \text { as } \delta \rightarrow 0 .
$$

If $0<\alpha \leq 1$ and $\omega(\delta)=\delta^{\alpha}$, we shall write $\Lambda_{\alpha}^{p}$ instead of $\Lambda(p, \omega)$, that is, we set

$$
\Lambda_{\alpha}^{p}=\Lambda\left(p, \delta^{\alpha}\right), \quad 0<\alpha \leq 1, \quad 1 \leq p \leq \infty .
$$

A classical result of Hardy and Littlewood [20] (see also Chapter 5 of [9]) asserts that for $1 \leq p \leq \infty$ and $0<\alpha \leq 1$, we have that

$$
\Lambda_{\alpha}^{p}=\left\{f \text { analytic in } \mathbb{D}: M_{p}\left(r, f^{\prime}\right)=\mathrm{O}\left(\frac{1}{(1-r)^{1-\alpha}}\right), \quad \text { as } r \rightarrow 1\right\} .
$$

Blasco and de Souza found in [6] conditions on $\omega$ so that the result of Hardy and Littlewood can be extended to the spaces $\Lambda(p, \omega)$. Namely, they proved that if $\omega$ satisfies the so called Dini condition and the condition $b_{1}$ (see $[\mathbf{6}],[\mathbf{5}]$ or $[\mathbf{1 3}]$ for the precise definitions) then, for $1 \leq p \leq \infty$,

$$
\Lambda(p, \omega)=\left\{f \text { analytic in } \mathbb{D}: M_{p}\left(r, f^{\prime}\right)=\mathrm{O}\left(\frac{\omega(1-r)}{1-r}\right), \text { as } r \rightarrow 1\right\} .
$$

Let $1 \leq p \leq \infty$ and let $\phi$ be a non-negative function defined in $[0,1)$. We define $\mathcal{L}(p, \phi)$ as the space of all functions $f$ analytic in $\mathbb{D}$ for which

$$
M_{p}\left(r, f^{\prime}\right)=\mathrm{O}(\phi(r)), \quad \text { as } r \rightarrow 1 .
$$

We remark that $\mathcal{L}(p, \phi) \subset \mathcal{L}(q, \phi)$, if $1 \leq q<p$. Notice that $(2.2)$ can be written as

$$
\Lambda_{\alpha}^{p}=\mathcal{L}\left(p, \frac{1}{(1-r)^{1-\alpha}}\right), \quad 1 \leq p \leq \infty, \quad 0<\alpha \leq 1,
$$

and (2.3) is equivalent to

$$
\Lambda(p, \omega)=\mathcal{L}\left(p, \frac{\omega(1-r)}{1-r}\right) .
$$

Cima and Petersen proved in $[\mathbf{8}]$ that $\Lambda_{1 / 2}^{2} \subset B M O A$. This result was extended by Bourdon, Shapiro and Sledd who proved the following result in [7]:

$$
\Lambda_{1 / p}^{p} \subset B M O A, \quad 1<p<\infty .
$$

For $p=1$ we have a stronger result. Indeed, a classical result of Privalov [9, Th. 3.11] asserts that a function $f$ which is analytic in $\mathbb{D}$ has a continuous extension to the closed unit disk $\overline{\mathbb{D}}$ whose boundary values are absolutely continuous on $\partial \mathbb{D}$ if and only if $f^{\prime} \in H^{1}$. In particular, we have

$$
f^{\prime} \in H^{1} \Rightarrow f \in \mathcal{A} \subset H^{\infty},
$$

where, $\mathcal{A}$ denotes the disk algebra. Using (2.2), we see that the the condition $f^{\prime} \in H^{1}$ is equivalent to saying that $f \in \Lambda_{1}^{1}$. Hence, (2.8) can be written as

$$
\Lambda_{1}^{1} \subset \mathcal{A} \subset H^{\infty} .
$$

The author proved in [12] that (2.8) (or, equivalently, (2.9)) is sharp in a very strong sense: 
TheOrem 2.1. ([12, Theorem 1]). Let $\phi$ be any positive continuous function defined in $[0,1)$ with $\phi(r) \rightarrow \infty$, as $r \rightarrow 1$. Then, there exists a function $f \in \mathcal{L}(1, \phi)$ which is not a normal function.

Hence, no condition on the growth of $M_{1}\left(r, f^{\prime}\right)$ other that its boundedness is enough to conclude that $f \in \mathcal{N}$. We recall here that $\mathcal{A} \subset H^{\infty} \subset B M O A \subset \mathcal{B} \subset \mathcal{N}$.

The original proof of Theorem 2.1 was constructive. The constructed function $f$ was of the form $f=B F$ where $B$ is a Blaschke product and $F$ is a function given by a series of analytic functions in $\mathbb{D}$ which converges uniformly on every compact subset of $\mathbb{D}$. The constructions of $B$ and $F$ were very involved, and made use in an essential way of certain sequences introduced by K. I. Oskolkov in several contexts (see, e.g., $[\mathbf{2 6}, \mathbf{2 7}, \mathbf{2 8}]$ ). But now is possible to give a much simpler proof of Theorem 2.1, independent of the Oskolkov's sequences, using Theorem 1.2 and the following result about Blaschke products proved in collaboration with C. González.

Theorem 2.2. ([15, Theorem 1]). Let $B$ be an interpolating Blaschke product with positive zeros. Then there exist two positive constants $C_{1}$ and $C_{2}$, and $\rho_{0} \in$ $(0,1)$ such that

$$
C_{1} n(r, B) \leq M_{1}\left(r, B^{\prime}\right) \leq C_{2} n(r, B), \quad \rho_{0}<r<1,
$$

where, for $0<r<1, n(r, B)$ denotes the number of zeros of $B$ in the disk $\{|z|<r\}$.

We remark that Girela and Peláez [18] have recently improved this result showing that the conclusion holds for any infinite Blaschke product whose sequence of zeros is exponential.

Proof of Theorem 2.1. Set $\varphi(r)=\sqrt{\phi(r)}, 0<r<1$. Using Theorem 1.2, we see that there exists a decreasing sequence of positive numbers $\Gamma=\left\{\gamma_{n}\right\}_{n=1}^{\infty}$ with $\gamma_{1}<1$ such that, if $F_{\Gamma}$ is the conformal mapping from $\mathbb{D}$ onto $\Omega_{\Gamma}$ with $F_{\Gamma}(0)=0$ and $F_{\Gamma}^{\prime}(0)>0$, then we have that $F_{\Gamma}(r) \rightarrow \infty$, as $r \rightarrow 1$ and

$$
M_{\infty}\left(r, F_{\Gamma}\right)=F_{\Gamma}(r) \leq \varphi(r), \quad 0<r<1 .
$$

Using the results of Section 1.5, it follows that we also have

$$
M_{1}\left(r, F_{\Gamma}^{\prime}\right)=\mathrm{O}(\varphi(r)), \quad \text { as } r \rightarrow 1 .
$$

Now, using Theorem 2.2, it follows easily (see $[\mathbf{1 5}$, pp. $7-8]$ for the details) that there exists an interpolating Blaschke product $B$ whose zeros $\left\{a_{n}\right\}$ are positive and such that

$$
M_{1}\left(r, B^{\prime}\right)=\mathrm{O}(\varphi(r)), \quad \text { as } r \rightarrow 1 .
$$

For simplicity set $F_{\Gamma}=F$ and define $f(z)=B(z) F(z)(z \in \mathbb{D})$. Bearing in mind that $B$ is bounded by 1 and using (2.12), (2.13) and (2.11), we see that

$$
M_{1}\left(r, f^{\prime}\right) \leq M_{1}\left(r, F^{\prime}\right)+M_{1}\left(r, B^{\prime}\right) M_{\infty}(r, F)=\mathrm{O}\left(\varphi^{2}(r)\right), \quad \text { as } r \rightarrow 1 .
$$

Hence, $f \in \mathcal{L}(1, \phi)$.

On the other hand, we have

$$
\left(1-\left|a_{n}\right|^{2}\right) \frac{\left|f^{\prime}\left(a_{n}\right)\right|}{1+\left|f\left(a_{n}\right)\right|^{2}}=\left(1-\left|a_{n}\right|^{2}\right)\left|B^{\prime}\left(a_{n}\right)\right|\left|F\left(a_{n}\right)\right| .
$$

Since $B$ is an interpolating Blaschke product, it follows that there exists $\delta>0$ such that $\left(1-\left|a_{n}\right|^{2}\right)\left|B^{\prime}\left(a_{n}\right)\right| \geq \delta$, for all $n$. The fact that $F$ is unbounded together with (2.11) implies that $F\left(a_{n}\right) \rightarrow \infty$, as $n \rightarrow \infty$. Then (2.14) implies that $\left(1-\left|a_{n}\right|^{2}\right) \frac{\left|f^{\prime}\left(a_{n}\right)\right|}{1+\left|f\left(a_{n}\right)\right|^{2}} \rightarrow \infty$, as $n \rightarrow \infty$ and, hence, $f$ is not a normal function. 
Similar arguments can be used to prove the sharpness of (2.7). Indeed, we have:

Theorem 2.3. ([15, Theorems 1.4 and 1.5]). Let $1 \leq p<\infty$ and let $\omega$ : $[0,1] \rightarrow[0, \infty)$ be a continuous and increasing function with $\omega(0)=0$ and

$$
\frac{\omega(\delta)}{\delta^{1 / p}} \rightarrow \infty, \quad \text { as } \delta \rightarrow 0 .
$$

and set $\phi(r)=\omega(1-r) /(1-r)(0<r<1)$, then there exists $f \in \mathcal{L}(p, \phi)$ which is not a normal function.

If we assume in addition that $\omega$ is a Dini weight and satisfies the condition $b_{1}$ then we can assert that there exists $f \in \Lambda(p, \omega)$ which is not a normal function.

The functions $f$ constructed to prove this theorem are also of the form $f=F B$ where $F$ is a conformal mapping from $\mathbb{D}$ onto one of the domains $\Omega_{\Gamma}$ considered in Section 1.5 and $B$ can be taken to be the Blaschke product whose sequence of zeros is $\left\{1-2^{-n}\right\}_{n=1}^{\infty}$.

2.3. An open question. All the examples of non-normal functions $f$ constructed in $[\mathbf{5}, \mathbf{1 2}, \mathbf{1 3}, \mathbf{1 5}]$ to prove the sharpness of $(2.7)$ and $(2.9)$ are of the form $f=F B$ where $F$ is a certain analytic function and $B$ is a Blaschke product. It is natural to ask whether or not Blaschke products are really needed. More precisely, we may raise the following questions.

Question 2.4. If $\phi$ is a positive continuous function defined in $[0,1)$ with $\phi(r) \rightarrow \infty$, as $r \rightarrow 1$, does there exist an outer function $f \in \mathcal{L}(1, \phi)$ which is not a normal function?

Question 2.5. Suppose that $1 \leq p<\infty$ and let $\omega:[0,1] \rightarrow[0, \infty)$ be a continuous and increasing function with $\omega(0)=0$ and $\omega(\delta) \delta^{-1 / p} \rightarrow \infty$, as $\delta \rightarrow 0$. Set $\phi(r)=\omega(1-r) /(1-r)(0<r<1)$. Does there exist an outer function $f \in \mathcal{L}(p, \phi)$ which is not a normal function?

We do not know the answer to these questions. We can just prove that there exist non-Bloch outer functions in the desired spaces $\mathcal{L}(p, \phi)$.

THEOREM 2.6. (i) Let $\phi$ be a positive continuous function defined in $[0,1)$ with $\phi(r) \rightarrow \infty$, as $r \rightarrow 1$. Then, there exists an outer function $f \in \mathcal{L}(1, \phi)$ which is not a Bloch function.

(ii) Suppose that $1 \leq p<\infty$ and let $\omega:[0,1] \rightarrow[0, \infty)$ be a continuous and increasing function with $\omega(0)=0$ and $\omega(\delta) \delta^{-1 / p} \rightarrow \infty$, as $\delta \rightarrow 0$. Set $\phi(r)=$ $\omega(1-r) /(1-r)(0<r<1)$. Then there exists an outer function $f \in \mathcal{L}(p, \phi)$ which is not a Bloch function

Proof. We shall just prove (i), the same argument works for (ii). Let $F$ and $B$ be the functions constructed in the proof of Theorem 2.1. Set $G(z)=F(z) / z(z \in \mathbb{D})$. Since $F$ is a conformal mapping from $\mathbb{D}$ onto a certain domain $\Omega$ with $F(0)=0$, it follows that $G$ is an outer function (see [9, Theorem 3.17]). Also, it is clear that

$$
M_{\infty}(r, G) \asymp M_{\infty}(r, F) \quad \text { and } \quad M_{1}\left(r, G^{\prime}\right) \asymp M_{1}\left(r, F^{\prime}\right), \quad \text { as } r \rightarrow 1 .
$$

On the other hand, $\operatorname{Re}(B(z)+1)>0$, for all $z$, which implies that $B+1$ is also an outer function. Since the product of two outer functions is an outer function, it 
follows that the function $g=G(B+1)$ is outer. Using (2.15), it follows easily that $g \in \mathcal{L}(1, \phi)$.

Recall that $B$ is an interpolating Blaschke product with positive zeros $\left\{a_{n}\right\}$ and that $F \in B M O A \subset \mathcal{B}$. Then, also $G \in \mathcal{B}$ and

$$
\left(1-\left|a_{n}\right|\right)\left|G^{\prime}\left(a_{n}\right)\left(B\left(a_{n}\right)+1\right)\right|=\left(1-\left|a_{n}\right|\right)\left|G^{\prime}\left(a_{n}\right)\right|=\mathrm{O}(1) .
$$

Since $\left(1-\left|a_{n}\right|\right)\left|B^{\prime}\left(a_{n}\right)\right| \geq \delta, n \geq 1$, for a certain $\delta>0$, and $\left|G\left(a_{n}\right)\right| \rightarrow \infty$, as $n \rightarrow \infty$, it follows that $\left(1-\left|a_{n}\right|\right)\left|B^{\prime}\left(a_{n}\right) G\left(a_{n}\right)\right| \rightarrow \infty$, as $n \rightarrow \infty$, which, together with (2.16), yields $\left(1-\left|a_{n}\right|\right)\left|g^{\prime}\left(a_{n}\right)\right| \rightarrow \infty$, as $n \rightarrow \infty$. Thus, $g \notin \mathcal{B}$.

\section{Bloch functions and division by inner functions}

In this section we shall consider another problem on analytic function spaces which can be solved making use of the conformal mappings introduced in Section 1.5.

We recall that a function $I$, analytic in $\mathbb{D}$, is said to be an inner function if $I \in H^{\infty}$ and $I$ has a radial limit $I\left(e^{i \theta}\right)$ of modulus one for almost every $e^{i \theta} \in \partial \mathbb{D}$.

Definition 3.1. A subspace $X$ of $H^{1}$ is said to have the $f$-property (or property of division by inner functions) if $h / I \in X$ whenever $h \in X$ and $I$ is an inner function with $h / I \in H^{1}$.

These notion was introduced by Havin [21]. Lots of spaces including the Hardy spaces $H^{p}(1 \leq p \leq \infty)$, the Dirichlet space $\mathcal{D}$ and several spaces of Dirichlet type, the spaces $B M O A$ and $V M O A$, the Lipschitz spaces $\Lambda_{\alpha}(0<\alpha \leq 1)$, the disc algebra $\mathcal{A}$ and many other are known to have the $f$-property. We mention the recently published paper $[\mathbf{1 7}]$ as a source to find these and many other results and references about the $f$-property and the stronger $K$-property introduced by Korenblum in $[\mathbf{2 5}]$.

The first example of a space not possessing the $f$-property was given by Gurarii $[\mathbf{1 9}]$ who proved that the space of analytic functions in $\mathbb{D}$ with an absolutely convergent power series does not have the $f$-property. Later on Anderson proved in $[\mathbf{1}]$ that $\mathcal{B}_{0} \cap H^{\infty}$ does not have the $f$-property. Consequently, the same is true for $\mathcal{B}_{0} \cap H^{p}$ for every $p \in[1, \infty)$.

Since $H^{\infty} \subset \mathcal{B}$, we see that $\mathcal{B} \cap H^{\infty}=H^{\infty}$ which has the $f$-property. However, for $1 \leq p<\infty$ the question of whether or not the space $\mathcal{B} \cap H^{p}$ has the $f$-property remained open. In a joint work with C. González and J. A. Peláez (cf. [16]) we have solved this problem, proving the following result:

Theorem 3.2. ([16, Theorem 1.4]). If $1 \leq p<\infty$ then the space $\mathcal{B} \cap H^{p}$ does not have the $f$-property.

Sketch of the proof. Let $B$ be an infinite Blaschke product in $\mathcal{B}_{0}$, whose sequence of zeros has a subsequence accumulating at 1 (the existence of such a product was first proved by Sarason [30]). Set

$$
\mu(r)=\sup _{r \leq|z|<1}\left(1-|z|^{2}\right)\left|B^{\prime}(z)\right|, \quad \varphi(r)=\frac{1}{\mu(r)}, \quad 0<r<1 .
$$

Since $B \in \mathcal{B}_{0}$, we have that $\varphi(r) \uparrow \infty$, as $r \uparrow 1$. Let $F=F_{\Gamma}$ be the conformal mapping constructed in Theorem 1.2 for this $\varphi$ and set $f=B F$. It is easy to see 
that

$$
f \in \mathcal{B} \cap H^{p}, \quad \text { for all } p<\infty .
$$

Let now $B_{1}$ be an interpolating Blaschke product, subproduct of $B$, whose sequence of zeros tends to 1 and set

$$
B_{2}=\frac{B}{B_{1}}, \quad g=\frac{f}{B_{2}}=B_{1} F .
$$

It is clear that $B_{2}$ is a Blaschke product and that $g \in H^{p}$ for all $p<\infty$. On the other hand, using that $B_{1}$ is an interpolating Blaschke product, we can deduce that $g \notin \mathcal{B}$ which, together with (3.1) and (3.2), implies that $\mathcal{B} \cap H^{p}(1 \leq p<\infty)$ does not have the $f$-property.

\section{Acknowledgements}

The author wishes to thank the referee for his/her comments and remarks.

\section{References}

[1] J. M. Anderson, On division by inner factors, Comment. Math. Helv. 54, 2 (1979), 309-317.

[2] J. M. Anderson, J. Clunie and Ch. Pommerenke, On Bloch functions and normal functions, J. Reine Angew. Math. 270 (1974), 12-37.

[3] A. Baernstein II, Integral means, univalent functions and circular symmetrization, Acta Math. 133 (1974), 139-169.

[4] A. Baernstein II, Analytic functions of bounded mean oscillation, in Aspects of Contemporary Complex Analysis, D. Brannan and J. Clunie (editors), Academic Press (1980), 3-36.

[5] O. Blasco, D. Girela and M. A. Márquez, Mean growth of the derivative of analytic functions, bounded mean oscillation, and normal functions, Indiana Univ. Math. J. 47 (1998), 893-912.

[6] O. Blasco and G. Soares de Souza, Spaces of analytic functions on the disc where the growth of $M_{p}(F, r)$ depends on a weight, J. Math. Anal. Appl. 147, 2 (1990), 580-598.

[7] P. Bourdon, J. Shapiro and W. Sledd, Fourier series, mean Lipschitz spaces and bounded mean oscillation, in Analysis at Urbana 1, Proceedings of the Special Year in Modern Analysis at the University of Illinois, 1986-87, E. R. Berkson, N. T. Peck and J. Uhl (editors), London Math. Soc. Lecture Notes Series 137, Cambridge Univ. Press (1989), 81-110.

[8] J. A. Cima and K. E. Petersen, Some analytic functions whose boundary values have bounded mean oscillation, Math. Z. 147 (1976), 237-347.

[9] P. L. Duren, Theory of $H^{p}$ Spaces, Academic Press, New York-London (1970). Reprint: Dover, Mineola, New York (2000).

[10] P. L. Duren, Univalent Functions, Springer Verlag, New York, Berlin, Heidelberg, Tokyo, (1983).

[11] J. B. Garnett, Bounded Analytic Functions, Academic Press, New York, etc. (1981).

[12] D. Girela, On a theorem of Privalov and normal funcions, Proc. Amer. Math. Soc. 125, no. 2 (1997), 433-442.

[13] D. Girela, Mean Lipschitz spaces and bounded mean oscillation, Illinois J. Math. 41, No. 2 (1997), 214-230.

[14] D. Girela, Analytic functions of bounded mean oscillation, in Complex functions spaces, $R$. Aulaskari (editor), Univ. Joensuu Dept. Math. Report Series No. 4 (2001), 61-171.

[15] D. Girela and C. González, Mean growth of the derivative of infinite Blaschke products, Complex Variables Theory Appl. 45, No. 1 (2001), 1-10.

[16] D. Girela, C. González and J. A. Peláez, Multiplication and division by inner functions in the space of Bloch functions, to appear in Proc. Amer. Math. Soc.

[17] D. Girela, C. González and J. A. Peláez, Toeplitz operators and division by inner functions, in Proceedings of the First Advanced Course in Operator Theory and Complex Analysis, A. Montes-Rodríguez (editor), Secretariado de Publicaciones de la Universidad de Sevilla (2005), 85-103. 
[18] D. Girela and J. A. Peláez, On the derivative of infinite Blaschke products, Illinois. J. Math. 48, No. 1 (2004), 121-130.

[19] V. P. Gurarii, The factorization of absolutely convergent Taylor series and Fourier integrals. (in Russian). Investigations on linear operators and the theory of functions, III, Zap. Naučn. Sem. Leningrad. Otdel. Mat. Inst. Steklov. (LOMI) 30 (1972), 15-32.

[20] G. H. Hardy and J. E. Littlewood Some properties of fractional integrals, II, Math. Z. 34 (1932), 403-439.

[21] V. P. Havin, On the factorization of analytic functions smooth up to the boundary (in Russian), Zap. Nauch. Sem. LOMI. 22 (1971), 202-205.

[22] W. K. Hayman, On functions with positive real part, J. London Math. Soc. 36 (1961), 35-48.

[23] J. A. Jenkins On circularly symmetric functions, Proc. Amer. Math. Soc. 6 (1955), 620-624.

[24] F. R. Keogh, Some theorems on conformal mapping of bounded star-shaped domains, Proc. London Math. Soc. 3, 9 (1959), 481-491.

[25] B. I. Korenblum, An extremal property of outer functions (in Russian), Mat. Zamet. 10 (1971), 53-56; translation in Math. Notes 10 (1971), 456-458 (1972).

[26] K. I. Oskolkov, Uniform modulus of continuity of summable functions on sets of positive measure, (in russian), Dokl. Akad. Nauk SSSR 229, 2 (1976), 304-306; English transl. in Sov. Math. Dokl. 17, 4 (1976/1977), 1028-1030.

[27] K. I. Oskolkov, Approximation properties of summable functions on sets of full measure, (in russian), Mat. Sbornik, n. Ser. 32, 4 (1977), 563-589; English transl. in Math. USSR Sbornik 32, 4 (1978), 489-514.

[28] K. I. Oskolkov, On Luzin's C-property for a conjugate function, (in russian), Trudy Mat. Inst. Stelklova, 164 (1983), 124-135; English transl. in Proc. Steklov Inst. Math. 164, (1985), 141153.

[29] Ch. Pommerenke, Univalent Functions, Vandenhoeck und Ruprecht, Göttingen, (1975).

[30] D. Sarason, Blaschke products in $\mathcal{B}_{0}$, Linear and Complex Analysis problem Book, Lecture Notes in Math. 1043 (Springer-Verlag, Berlin, Heidelberg, New York, Tokyo 1984), 337-338.

Departamento de Análisis Matemático, Facultad de Ciencias, Universidad de Málaga, 29071 Málaga, Spain

E-mail address: girela@uma.es 